\title{
Interdiffusion in dilute polymer mixtures. A subtle concentration effect
}

\author{
T. Jian, S. H. Anastasiadis, ${ }^{\text {a) }}$ A. K. Rizos, ${ }^{\text {b) }}$ and G. Fytas ${ }^{\text {c) }}$ \\ Foundation for Research and Technology-Hellas, Institute of Electronic Structure and Laser; P.O. Box 1527, \\ 711 10 Heraklion Crete, Greece
}

\begin{abstract}
A. Z. Akcasu
Department of Nuclear Engineering, University of Michigan, Ann Arbor, Michigan 48109
\end{abstract}

(Received 25 October 1993; accepted 25 April 1994)

\begin{abstract}
Dynamic light scattering has been used to investigate the diffusional dynamics in very dilute polystyrene/poly(propylene oxide), PS/PPO, polymer blends. Compared to previous investigations in the field, this system is more suitable for this type of investigation due to the significant refractive index difference between the two components and the fact that the matrix (PPO) dynamics do not interfere with the measurements. The tracer diffusion coefficient of PS thus obtained in the limit of infinite dilution scales as $N_{\mathrm{PS}}^{-0.8 \pm 0.04}$ with the PS degree of polymerization, i.e., behavior intermediate between the limits of nondraining Zimm and free-draining Rouse behavior. The effect of the addition of a third component even at tracer concentrations on the diffusion dynamics was investigated both experimentally and theoretically in the framework of the dynamic random phase approximation. Similarities and differences between theory and experiment were found that are rather due to a modification of hydrodynamic interactions.
\end{abstract}

\section{INTRODUCTION}

There exist an abundance of data on translational diffusion in dilute polymer solutions. ${ }^{1}$ The limiting low polymer concentration diffusion coefficient, $D^{0}$, is dominated by nondraining hydrodynamic effects and the dependence of $D^{0}$ on the degree of polymerization $N$ can be described by the exponential law

$$
D^{0} \propto N^{-\nu} \text {. }
$$

For $N>300$, the exponent $\nu=\overline{0} .51$ and 0.58 for theta and good solvents, respectively. For shorter chains deviations from this scaling behavior were reported in both good and theta solvents, probably arising from free-draining contributions. ${ }^{2,3}$ In the dilute regime with polymer concentration, $c$, less than the overlap concentration, $c^{*}$, an increase of $c$ causes the diffusion coefficient, $D$, to cithcr decrease or increase depending on the solvent quality. The linear concentration dependence

$$
D=D^{0}\left(1+k_{D} c\right)
$$

has been documented by dynamic light scattering (DLS) from polymer solutions with $N$ ranging from 12 to $2 \times 10^{4}$ and $c / c^{*}$ as low as 0.02 . The interaction parameter $k_{D}$ depends on $N$ and is negative for "theta" and positive for "good" solvents.

Polymer diffusion behavior in dilute polymer blends (A/B) where $\mathrm{B}$ is a polymeric solvent may be quite different. The polymeric "solvent" molecules become now entropic springs, which may affect the hydrodynamic flow of the minority chains A and, hence, the exponent in Eq. (1). Moreover, thermodynamic interactions can affect the conforma-

\footnotetext{
a)Also at University of Crctc, Physics Department, 71110 Heraklion Crete, Greece.

b) Also at University of Crete, Chemistry Department, 71409 Heraklion Crete, Greece.

c) Author to whom correspondence should be addressed.
}

tion of chains A as shown by computer simulations for a dilute binary polymer mixture. ${ }^{4}$ The predicted coil contraction with increasing interaction strength would cause faster diffusion and change the slope in Eq. (2). Besides, the underlying assumptions, related to the glass transition, in the theoretical treatments ${ }^{5}$ of the interdiffusion in binary polymer mixtures at finite compositions can rather be fulfilled ${ }^{6}$ at low concentrations. In the last years, the experimental effort on diffusional dynamics of polymer blends at finite concentrations is significantly reduced mainly because of the difficulties faced in the analysis of the concentration dependence of the interdiffusion data. ${ }^{6}$ However, it has been established ${ }^{7}$ that $D$ in unentangled polymer blends exhibits a freedraining Rouse behavior $(\nu=1)$, assumed by the theories of polymer interdiffusion. This dependence was also supported by a subsequent study of dilute polystyrene/poly(methylphenyl-siloxane), PS/PMPS, blends ${ }^{8}$ for which the first dynamic light scattering report ${ }^{9}$ erroneously claimed nondraining $(\nu \cong 0.5)$ conditions. A severe deficiency of the latter system was the presence of dynamic light scattering from the bulk PMPS sample, arising from concentration fluctuations due to the presence of oligomers, and the moderate refractive index difference $(\delta n=0.06)$ between the blend components that precluded measurements at very low concentrations.

In the present paper, we employ photon correlation spectroscopy (PCS) in the polarized geometry to investigate the diffusional dynamics in polystyrene/poly(propylene oxide), $\mathrm{PS} / \mathrm{PPO}$, blends at $c / c^{*}$ ranging between $5 \times 10^{-2}$ and $2.5 \times 10^{-3}$ at $25^{\circ} \mathrm{C}$. The solvent PPO has $N_{\mathrm{PPO}}=35$, whereas four different PS molecular weights were employed with $N_{\text {PS }}$ between 17 and 160. This system possesses a refractive index difference $\delta n=0.15$ while the PPO displays only very fast dynamic light scattering due to density fluctuations. The variation of the diffusion coefficient, $D$, with blend composition and $N_{\mathrm{PS}}$ in dilute binary PS/PPO blends is discussed in Sec.IV. The PS tracer diffusion coefficient is found to scale as $N_{\mathrm{PS}}^{-0.8 \pm 0.04}$, a behavior between the nondraining Zimm and 
the free-draining Rouse behavior. In fact, it was found that the PS translational diffusion is very sensitive to the presence of a third component even at tracer concentrations, and, in order to verify the random phase approximation predictions and the possible effect of hydrodynamic interactions, we have extended the sudy to ternary PS/S/PPO systems, with S being a low molecular weight component isorefractive either with the matrix PPO $\left(\mathrm{S} \equiv \mathrm{CCl}_{4}\right)$ or with the minority solute PS ( $\mathrm{S} \equiv 1,3$-dibromobenzene).$^{10}$ It should be mentioned that all the ternary polymer solutions investigated so far by PCS were in the semidilute regime. ${ }^{11-13}$ The intermediate dynamic structure factor for these dilute ternary systems is calculated in Sec. II in the framework of the dynamic random phase approximation, ${ }^{14}$ and the new results are discussed in relation to the theory. Theory qualitatively captures the pertinent features of the experimental observations, whereas deviations from the theoretical predictions are discussed in terms of hydrodynamic interactions in Sec. V.

\section{THEORY}

The general theory for the calculation of the intermediate dynamic structure factor in multicomponent systems was first formulated by Akcasu et al. ${ }^{14}$ within the framework of random phase approximation (RPA). The spirit of this approach is to express the total intensity scattered by various species in the interacting system in terms of scattered intensity by the individual molecules. The approach has been since used to investigate the dynamical properties for a variety of polymer systems in bulk ${ }^{15}$ and in solution. ${ }^{16}$ The general expressions for the dynamic scattering function and its first cumulant in an interacting system for multicomponent incompressible polymer mixtures have recently appeared. ${ }^{17}$ We expand this approach for the case of an interacting system of two polymers and a small amount of a third component treated as polymeric. The applicability of RPA when a small molecule is involved may be questionable, but for the concentrated regime under discussion here, mean field may be applied.

For an incompressible ternary system, the intermediate structure factor from a labeled component, $\mathrm{A}$, in a mixture with $\mathrm{B}$ and $\mathrm{C}$ with scattering lengths $b=c$ can be expressed as $^{14}$

$$
S_{a a}(q, t)=a_{1}(q) \exp \left[-\lambda_{1}(q) t\right]+a_{2}(q) \exp \left[-\lambda_{2}(q) t\right]
$$

where $\lambda_{1}(q)$ and $\lambda_{2}(q)$ are the eigenvalues of the first cumulant matrix ${ }^{14,17}$ and $\alpha_{1}(q)$ and $\alpha_{2}(q)$ are the respective amplitudes. If it is assumed that the minority component $\mathrm{C}$ does not interact with either $\mathrm{A}$ or $\mathrm{B}$, i.e., for the interaction parameters $\chi_{a c}=\chi_{b c}=0$ and $\chi_{a b}=\chi$, the RPA formalism for the three different situations that correspond to the three systems investigated in this work leads to the following.

\section{A. Two component dilute mixture (system 0 )}

For the limit of low third component concentration, $\phi_{c} \rightarrow 0$, i.e., for a two component interacting system, this approach correctly reproduces a single relaxation process that corresponds to the interdiffusion in $\mathrm{A} / \mathrm{B}$ blends, ${ }^{14}$ with diffu- sion coefficient $\lambda_{1} /\left(q^{2} k_{\mathrm{B}} T\right)=D_{\text {in,bulk A-B }}$. In the dilute regime $\left(\phi_{a} \rightarrow 0\right)$, the resulting diffusion coefficient is

$$
\begin{aligned}
D_{\phi_{c}=0} \rightarrow & D_{a}^{0}\left[1-\left(\frac{N_{a} D_{a}^{0}}{N_{b} D_{b}^{0}}-\frac{N_{a}}{N_{b}}+2 \chi N_{a}\right) \phi_{a}\right], \\
& \phi_{a} \rightarrow 0, \quad \phi_{c}=0, \\
a_{\phi_{c}=0} \rightarrow & \phi_{a} N_{a}, \quad \phi_{a} \rightarrow 0, \quad \phi_{c}=0,
\end{aligned}
$$

where $D_{i}$ 's are the tracer diffusion coefficients, which in the Rouse regime are given by $D_{i}=k_{\mathrm{B}} T / N_{i} \zeta_{i}$ with $N_{i}$ the number of monomers per chain and $\zeta_{i}$ the friction coefficients. For $N_{a}=N_{b}=N$ and $D_{a}^{0}=D_{b}^{0}=D^{0}$, this can be written as

$$
D_{\varphi_{c}=0} \rightarrow D_{a}^{0}\left(1-2 \chi N_{a} \phi_{a}\right), \quad \phi_{a} \rightarrow 0, \quad \phi_{c}=0
$$

with $\phi_{a}=\phi_{\mathrm{PS}}$, in agreement with the functional form of Eq. (2). This shows that the interdiffusion at very low volume fractions coincides with the tracer diffusion coefficient of the minority chains, and, hence, it should exhibit an $N^{-1}$ molecular weight dependence if $D_{a}^{0}=k_{\mathrm{B}} T / N_{a} \zeta_{a}$.

\section{B. Three component dilute mixture}

Two different limits should be explored for a three component system. System I corresponds to the case of the third component being isorefractive with the "solvent" chains, whereas in system II, the third component is assumed isorefractive with the minority component. In order to use the RPA formalism with A being the visible component and components $\mathrm{B}$ and $\mathrm{C}$ possessing equal refractive indices, we identify the three components as follows: system I corresponds to $\mathrm{A} \equiv$ tracer $\left(\phi_{a} \rightarrow 0\right), \mathrm{B} \equiv$ matrix, $\mathrm{C} \equiv$ third component, whereas system II exhibits $\mathrm{A} \equiv$ matrix, $\mathrm{B} \equiv$ tracer $\left(\phi_{b} \rightarrow 0\right)$, and $\mathrm{C} \equiv$ additive.

The theory for the case of $N_{a}=N_{b}=N$ and $D_{a}^{\mathrm{I}}=D_{b}^{\mathrm{I}}$ $=D^{\mathrm{I}}$ predicts:

(I) for system I and for $\phi_{a} \rightarrow 0$

$$
\begin{aligned}
& D_{1}^{\mathrm{I}} \rightarrow D_{c}^{\mathrm{I}} D^{\mathrm{I}} \frac{N\left(1-\phi_{c}\right)+\phi_{c} N_{c}-2 \chi N N_{c} \phi_{c} \phi_{a}}{\left(1-\phi_{c}\right) N D^{\mathrm{I}}+\phi_{c} N_{c} D_{c}^{\mathrm{I}}}, \\
& \phi_{a} \rightarrow 0 \\
& \ddot{a} \rightarrow 0, \quad \phi_{a} \rightarrow 0, \\
& D_{2}^{\mathrm{I}} \rightarrow D^{\mathrm{I}}\left[1-\left(2 \chi^{N}\right.\right. \\
& \left.\left.-\frac{\phi_{c} N_{c} D_{c}^{\mathrm{I}}}{\left(1-\phi_{c}\right)\left[\left(1-\phi_{c}\right) N D_{c}^{1}+\phi_{c} N_{c} D_{c}^{\mathrm{I}}\right]}\right) \phi_{a}\right] \\
& \rightarrow D^{\mathrm{I}}\left[1-2 \chi N \phi_{a}\right], \quad \phi_{a} \rightarrow 0, \\
& a_{2}^{\mathrm{I}} \rightarrow N \phi_{a}, \quad \phi_{a} \rightarrow 0,
\end{aligned}
$$

where $D^{\mathrm{I}}$ and $D_{c}^{\mathrm{I}}$ are the tracer diffusion coefficients in the multicomponent medium that depend on $\varphi_{b}$ and $\varphi_{c}$ via the friction coefficients.

(II) for system II and for $\phi_{b} \rightarrow 0$

$$
\begin{aligned}
D_{1}^{\mathrm{II}} & \rightarrow D_{c}^{\mathrm{II}} D^{\mathrm{II}} \frac{N\left(1-\phi_{c}\right)+\phi_{c} N_{c}-2 \chi N N_{c} \phi_{c} \phi_{b}}{\left(1-\phi_{c}\right) N D^{\mathrm{II}}+\phi_{c} N_{c} D_{c}^{\mathrm{II}}} \\
& \rightarrow D_{c}^{\mathrm{II}}, \quad \phi_{b} \rightarrow 0,
\end{aligned}
$$




$$
\begin{aligned}
& a_{1}^{\mathrm{I}} \rightarrow \frac{\phi_{c} N N_{c}}{\left(1-\phi_{c}\right) N+\phi_{c} N_{c}}\left[\left(1-\phi_{c}\right)-2 \phi_{b}\right], \quad \phi_{b} \rightarrow 0, \\
& D_{2}^{\mathrm{II}} \rightarrow D^{\mathrm{I}}\left[1-2 \chi N \phi_{b}\right], \quad \phi_{b} \rightarrow 0, \\
& a_{2}^{\mathrm{II}} \rightarrow N \phi_{b}, \quad \phi_{b} \rightarrow 0, \\
& \frac{a_{1}^{\mathrm{II}}}{a_{2}^{\mathrm{I}}} \rightarrow \frac{\phi_{c} N_{c}}{\left(1-\phi_{c}\right) N+\phi_{c} N_{c}}\left(-2+\frac{1-\phi_{c}}{\phi_{b}}\right), \quad \phi_{b} \rightarrow 0 .
\end{aligned}
$$

Again $D^{\mathrm{II}}$ and $D_{c}^{\mathrm{II}}$ depend on $\varphi_{a}$ and $\varphi_{c}$.

The qualitative differences predicted for the two cases examined are discussed, while keeping in mind the assumptions involved (treatment of component $\mathrm{C}$ as polymeric not interacting with either A or B). For system I, one diffusive relaxation mode is predicted for low concentrations of the tracer component with amplitude that increases linearly with the tracer volume fraction. The observed diffusion constant is the interdiffusion constant in $\Lambda$ and $(B+C)$, with intercept the tracer diffusion constant of $\mathrm{A}$ in the effective medium $(B+C)$. Comparing this case I with the binary mixture, similar dependencies are predicted for the diffusion coefficient and the corresponding amplitude as a function of $\phi_{a}$. The intercept $D^{I}$ should be slightly different than $D^{0}$ because of the change in the local friction by the presence of the third component $\mathrm{C}$, which is manifested as a change in the viscosity of the blend. However, the slope of $D_{2}^{\mathrm{I}} / D^{\mathrm{I}}$ vs $\phi_{a}$ should be the same as the $D_{\phi_{c}=0} / D^{0}$ vs $\phi_{a}$ in the binary mixture. For case II, two diffusive relaxation processes characterize the time evolution of the intermediate scattering function. The fast mode (mode 1) has an amplitude that is virtually insensitive to the tracer volume fraction, $\varphi_{b}$, whereas the amplitude of the slow mode depends linearly on $\varphi_{b}$. Moreover, the ratio of the amplitudes of the fast to the slow mode varies linearly with $1 / \varphi_{b}$. With respect to the diffusion constants, the slow mode resembles the active mode in case $\mathrm{I}$, i.e., it is the interdiffusion in $\mathrm{B} /(\mathrm{A}+\mathrm{C})$. As before, the intercept $D^{I}$ should be modified by the presence of $\mathrm{C}$ where the slope of the $D_{2}^{\Pi} / D^{\Pi}$ vs $\phi_{b}$ should be the same as in the binary mixture $D_{\phi_{c}=0} / D^{0}$ vs $\varphi_{a}$. The diffusion constant of the fast mode should have an amplitude that is essentially $D_{c}$, the tracer diffusion of $\mathrm{C}$ in the mixture, and very small slope of the $D_{1}^{\Pi} / D_{c}^{\Pi}$ vs $\varphi_{b}$ line of $-2 \chi N_{c} \varphi_{c}\left(1-\varphi_{c}\right)$.

\section{EXPERIMENT}

\section{A. Materials}

Poly(propylene oxide) (PPO) with $M_{n}=2025$ purchased from Merk was used as the matrix ("solvent") throughout the work presented here. The polystyrene (PS) samples used as the minority component were obtained from Pressure Chemicals. Four different molecular weights were used with $M_{w}=1700,3800,9000$, and 16700 and low polydispersity $M_{w} / M_{n}<1.05$. Spectral grade $\mathrm{CCl}_{4}(\mathrm{~T})$ and analytical grade 1,3-dibromobenzene (DB) were used as received as the third component additive in low quantities. In the visible spcctrum, the refractive index of $\mathrm{T}(n=1.45)$ and $\mathrm{DB}(n=1.60)$ are quite close to that of PPO (1.44) and PS (1.59), respectively.
A stock solution of PS/PPO with PS volume fraction $\phi_{\mathrm{PS}}=0.01$ was prepared by dissolving the PS component at $70^{\circ} \mathrm{C}$. All binary PS/PPO samples were then obtained by dilution of this initial solution with bulk PPO. Similarly we used a stock solution S/PPO ( $\mathrm{S}$ denotes $\mathrm{T}$ or $\mathrm{DB}$ ) with $\phi_{\mathrm{S}}=0.015$ to prepare all ternary PS/S/PPO mixtures by dilution of the same initial PS/S/PPO solution with $\phi_{\mathrm{PS}}=0.01$ and $\phi_{S}=0.015$. Dust-free samples were obtained by filtration through $0.22 \mu \mathrm{m}$ Millipore filters directly into the light scattering cylindrical cells $(12.7 \mathrm{~mm}$ o.d.). The quality of the samples will be evident by the stability of the trace of the light scattering intensity over the duration of the photon correlation measurements. More than 25 solutions were investigated.

\section{B. Photon correlation spectroscopy (PCS)}

The autocorrelation function $G_{V V}(q, t)$ of the polarized light scattering intensity was measured at various scattering angles, $\theta$ (from $30^{\circ}$ to $150^{\circ}$ ), using an ALV-5000 digital full correlator over the time range $10^{-6}-10^{3} \mathrm{~s}$. Both the incident beam with wavelength $\lambda=488 \mathrm{~nm}$ at a single mode intensity $100 \mathrm{~mW}$ and the scattered light were polarized perpendicular $(V)$ to the scattering plane. Under homodyne conditions, the desired normalized field correlation function $g(q, t)$, at a given scattering vector $q$, whose magnitude is equal to ( $4 \pi n$ / $\lambda) \sin (\theta / 2)$ with $n$ being the refractive index of the medium, is related to $G_{V V}(q, t)$ by

$$
G_{V V}(q, t)=A_{\infty}\left[1+f^{*}|a g(q, t)|^{2}\right],
$$

where $f^{*}$ is an instrumental factor obtained from the $G_{V V}(q, t)$ of a dilute PS/CCl 4 solution $\left(f^{*} \cong 0.7\right)$, $a$ is the fraction of the total polarized scattered intensity with correlation times longer than about $10^{-6} \mathrm{~s}$, and $A_{\infty}$ is the baseline measured at long delay times. Due to the low concentration of relatively low molecular weight PS, the light scattering intensity $I_{c}$ arising from concentration fluctuations is very low. Hence, the amplitude $a=I_{c} / I$ of $g(q, t)$ at short times $\left(<10^{-6} \mathrm{~s}\right)$ is expected and found (Fig. 1) to be less than one. $I$ is the total light scattering intensity $I=I_{\mathrm{PPO}}+I_{c}$ and $I_{\mathrm{PPO}}$ is the contribution due to the density fluctuations of the matrix PPO. For this reason, long accumulation times and extremely clean solutions are required, especially in the very dilute limit $\left(\phi \sim 10^{-3}\right)$.

\section{Viscosity measurements}

A Haake falling-ball viscometer was utilized to measure the viscosity of the various mixtures after calibration for the instrumental factor. The temperature was controlled within $25 \pm 0.1^{\circ} \mathrm{C}$ over the duration of the experiments.

\section{DATA ANALYSIS}

\section{A. PS/PPO and P/T/PPO}

The net experimental intensity autocorrelation functions $C(q, t) \equiv a g(q, t)$ for both PS/PPO binary and PS/T/PPO ternary systems are well represented by the KohlrauschWilliams-Watts (KWW) decay functions

$$
C(q, t)=a \exp \left[-(t / \tau)^{\beta}\right]+a^{\prime} .
$$




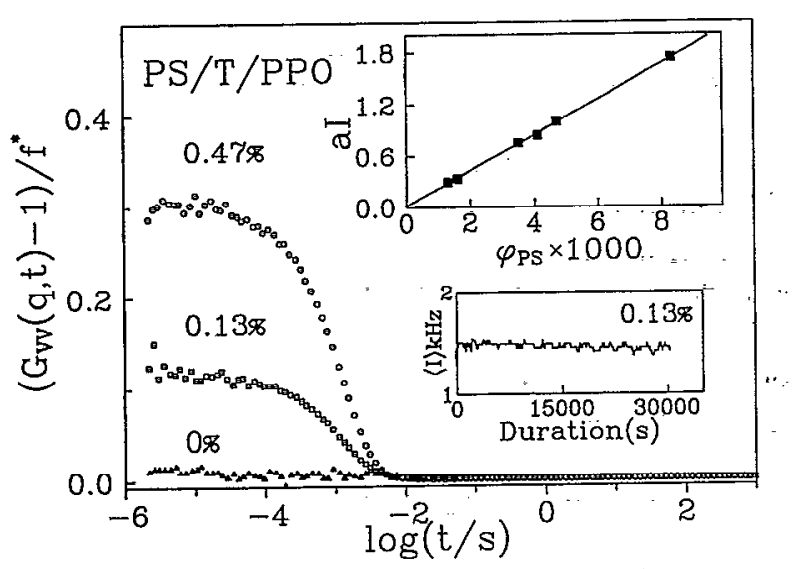

FIG. 1. Intensity correlation functions for polystyrene/CCl $/$ poly(propylene oxide) blends with $1.5 \% \mathrm{CCl}_{4}, N_{\mathrm{PS}}=35$ and three different PS compositions at $25^{\circ} \mathrm{C}$ and $90^{\circ}$ scattering angle. The insets display the intensity profile during the duration of the experiment for the $0.13 \%$ sample and the polystyrene concentration dependence of the light scattering intensity, $a\langle I\rangle$, due to concentration fluctuations. Note the featureless correlation function for the isorefractive $\mathrm{CCl}_{4} /$ poly (propylene oxide) solution $\left(\phi_{\mathrm{PS}}=0\right.$ ).

The value of the distribution parameter $\beta=0.96 \pm 0.03 \mathrm{im}$ plies almost exponential decay and the adjustable parameter $a^{\prime}$, accounting for small baseline fluctuations, is close to zero. Figure 1 displays experimental $C(q, t)$ for two PS/T/ PPO samples with $1.5 \% \mathrm{~T}, N_{\mathrm{PS}}=35$, and two different PS compositions at $25^{\circ} \mathrm{C}$ and $\theta=90^{\circ}$. The light scattering intensity profile throughout the duration of the experiment is displayed as inset in Fig. 1 for the $0.13 \%$ sample. This indicates the high optical quality of the samples and the absence of any additional slower process due to spurious scattering. The second inset of Fig. 1 provides further support of the experimental data and analysis since the average light scattering intensity $I_{\mathrm{PS}}=a\langle I\rangle$, being only a fraction of the total $\langle I\rangle$ intensity and due mainly to concentration fluctuations scales nicely with PS composition in this dilute regime. It is also evident from the featureless experimental correlation function of T/PPO solution (Fig. 1) that the translational dynamics of $\mathrm{T}$ is hardly observed due to the very small refractive index increment of the solution. The experimental $C(q, t)$ of the ternary PS/T/PPO system can, therefore, be described by the single exponential decay function of Eq. (9).

\section{B. PS/DB/PPO}

As expected and in agreement with Eq. (7), the experimental functions $C(q, t)$ for this system clearly show two distinct decays. Figure 2 illustrates this situation for three samples with $N_{\mathrm{PS}}=35,1.6 \% \mathrm{DB}$, and three different PS concentrations at $25^{\circ} \mathrm{C}$ and $\theta=90^{\circ}$. Based on the almost single exponential correlation function of the previous system, we have represented the experimental $C(q, t)$ of Fig. 2 with a double exponential function

$$
C(q, t)=a_{1} \exp \left(-t / \tau_{1}\right)+a_{2} \exp \left(-t / \tau_{2}\right)+a^{\prime},
$$

where $a_{1}, \tau_{1}$ and $a_{2}, \tau_{2}$ are the amplitude and the relaxation time for the fast and slow process, respectively. The uniqueness of the five parameter fit was examined by verifying that the ratio $a_{2} / a_{1}$ is proportional to $\phi_{\mathrm{PS}}$ for constant $\mathrm{DB}$ com-

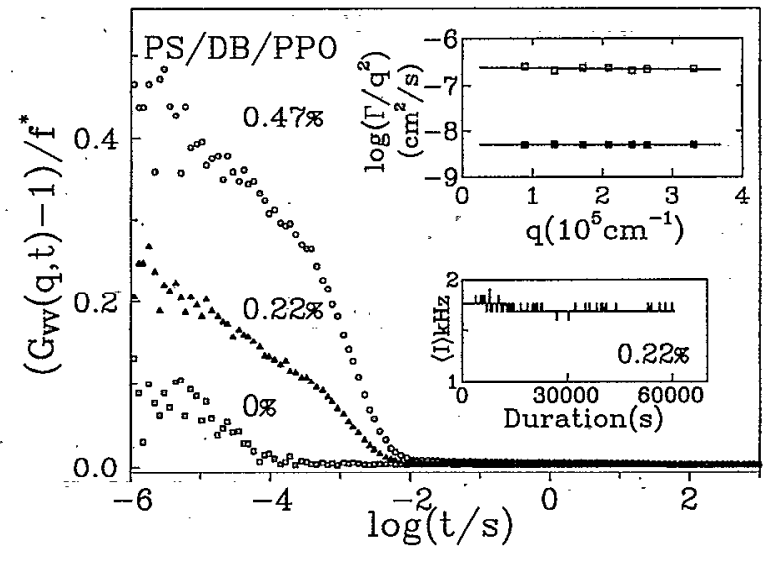

FIG. 2. Intensity correlation functions for three polystyrene/1,3dibromobenzene/poly(propylene oxide) mixtures with $1.6 \% \quad 1,3-$ dibromobenzene, $N_{\mathrm{PS}}=35$, at $25^{\circ} \mathrm{C}$ for three different polystyrene concentrations at $\theta=90^{\circ}$. The $q^{2}$ dependence of the two relaxation rates and the intensity trace for the $0.22 \%$ solution are shown in the inset. In contrast to the $\mathrm{CCl}_{4} /$ poly(propylene oxide) of Fig. 1, the binary 1,3-dibromobenzene/ poly(propylene oxide) solution $\left(\phi_{\mathrm{PS}}=0\right)$ displays dynamic light scattering due to finite refractive index increment.

position, and the closeness of the $\tau_{1}$ values with the relaxation times in DB/PPO solution with concentration 1.6\%. As expected, both relaxation rates $\Gamma_{1}$, and $\Gamma_{2}(\Gamma=1 / \tau)$ were found to be proportional to $q^{2}$ (inset of Fig. 2). Hence, the diffusion coefficients are computed by $D=\Gamma / q^{2}$ from PCS measurements at different scattering angles. Due to the dilute conditions, accumulations over 10-20 here necessary, that required very stable experimental conditions, reflected in the intensity trace (inset of Fig. 2) and the featureless correlation function of the bulk PPO (Fig. 3 below).

\section{RESULTS AND DISCUSSION}

\section{A. Dilute binary blends (PS/PPO)}

Figure 3 shows two net polarized intensity correlation

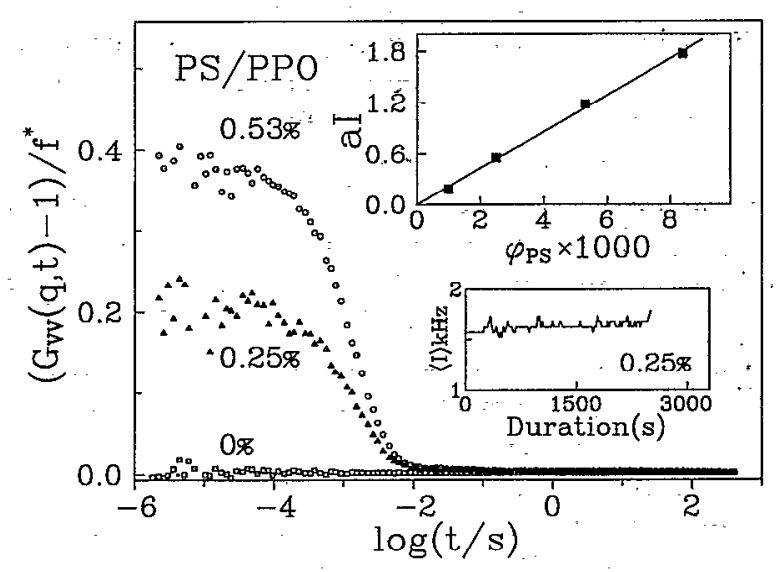

FIG. 3. Polarized intensity correlation functions for two polystyrene/ poly(propylene oxide) dilute blends and for a poly(propylene oxide) homopolymer at $25^{\circ} \mathrm{C}$ and $90^{\circ}$ scattering angle. The insets display the polystyrene concentration dependence of the dynamic light scattering intensity, $a\langle I\rangle$, and the intensity trace for the $0.25 \%$ mixture. 


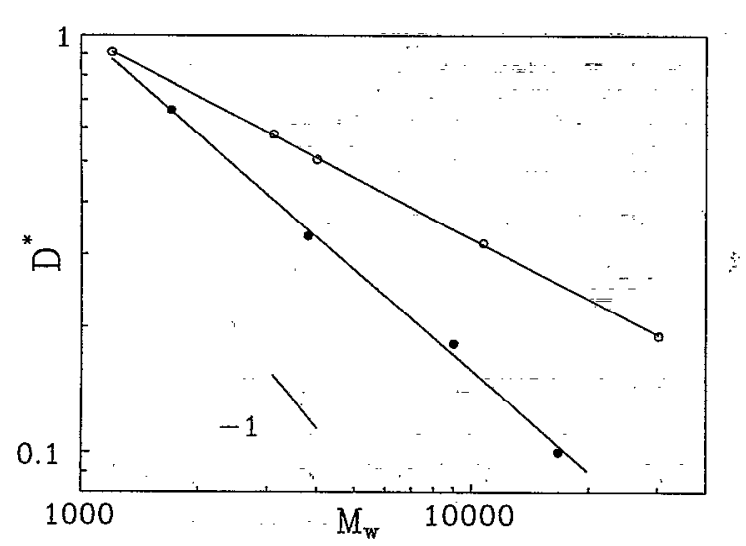

FIG. 4. The variation of the polystyrene tracer diffusion coefficient in the polystyrene/poly(propylene oxide) dilute binary blends at $25^{\circ} \mathrm{C}(0)$ and in polystyrene/cyclohexane (Ref. 3) at $34.5^{\circ} \mathrm{C}(\mathrm{O})$ with the PS molecular weight. The diffusion data are normalized to the respective values for polystyrene with molecular weight $1000\left(D=1.58 \times 10^{-8}\right.$ and $3.85 \times 10^{-6} \mathrm{~cm}^{2} / \mathrm{s}$, respectively). The solid line denotes the slope of -1 predicted for freedraining conditions.

functions due to composition fluctuations in the very dilute binary PS/PPO polymer mixtures; the lowest concentration is about 1000 times less than the overlap concentration $c^{*}$ for PS $(N=35)$. For the latter mixture, the dynamic light scattering arising from concentration fluctuations still amounts to about $25 \%$ of the total polarized intensity (including the contribution of the much faster density fluctuations). The featureless correlation function of Fig. 3 is for the bulk PPO homopolymer, that has no measurable effect on the experimental $G_{V V}(q, t)$ of the blends even for the lowest PS concentration. This situation should be contrasted with the PS/ PMPS dilute blends, ${ }^{8,9}$ for which the bulk PMPS displayed significant dynamic light scattering thus affecting the interdiffusion results.

The present blend appears to be a model system allowing the extension of interdiffusion measurements at very low PS concentrations. The dynamic intensity $I_{c}=a\langle I\rangle$ is predicted, Eq. (4b), and found to be proportional to $\phi_{\mathrm{PS}}$ (inset of Fig. 3) at low PS concentrations. This finding supports the neglect of the thermodynamic interactions $\left(2 \chi N \phi_{\mathrm{PS}}\right)$ and is in accord with the very weak (see Fig. 5 below) concentration dependence of the interdiffusion coefficient $D$. Under these conditions, the latter is essentially the tracer diffusion coefficient $D_{\mathrm{PS}}^{0}$ of the minority chains (PS), Eq. (5). It is, therefore, meaningful to examine the $N$ dependence of $D_{\mathrm{PS}}$ and compare it with the RPA prediction.

The experimental $D_{\mathrm{PS}}$ is found to scale with $N^{-0.8 \pm 0.04}$ as shown in Fig. 4. For comparison, the translational diffusion of PS in the molecular "theta" solvent cyclohexane, shown also in Fig. 4, scales with $N^{-0.48}$ over very similar range of molecular weights. ${ }^{3}$ The distinctly different diffusional behavior in the polymeric (PPO) solvent suggests an increasing domination of the screened (Rouse) hydrodynamic interactions.

Somewhat lower values of the exponent $\nu$ were reported for the tracer diffusion of labeled PPO chains (about $0.05 \%$ ) in unlabeled PPO matrix chains obtained from pattern fluo- rescence reappearance after photobleaching experiments. ${ }^{18}$ For a PPO matrix with $M_{w}=1960, D \propto N^{-0.6}$ whereas for PPO $M_{w}=4220, D \propto N^{-0.75}$, when the molecular weight of the labeled PPO tracer varied from 800 to 94000 . It appears that the hydrodynamic interactions decrease as the PPO matrix molecular weight increases. Moreover, revisiting the tracer diffusion data in dilute PS/PMPS blends ${ }^{8}$ and excluding the lowest molecular weight data results in a $D \propto N^{-0.74}$ dependence. Therefore, the polymeric nature of the solvent promotes free-draining conditions, similarly to the situation in polymer blends at finite concentrations. ${ }^{6,714}$ The dependence of the tracer diffusion coefficient on both tracer $(N)$ and matrix $(\bar{P})$ molecular weights were also investigated by forward-recoil spectroscopy ${ }^{19}$ for high molecular weight (55 000-915 000) deuterated PS tracer in PS matrices. For low matrix molecular weights $(<10000)$ in the unentangled regime, a $D \propto N^{-0.61} P^{-1}$ dependence has been observed, whereas, as the matrix molecular weight is increased, the intermediate constraint-release regime is observed followed by the reptation in a fixed environment of constraints regime with $D \propto N^{-2} P^{0}$. The $N^{-0.61}$ dependence for low $P$ was attributed to hydrodynamic interactions between segments of the tracer chain being unscreened for unentangled matrices with molecular weights lower than those of the diffusing species. In those experiments, the concentration of the tracer was about $10 \%$ and changes in the mobilities near the glass transition temperature $\left(T_{g}\right)$ arising from the molecular weight dependence of $T_{g}$ were accounted for by comparing the data at constant fractional free volume. Such correction is unnecessary in the present PCS and earlier photoblcaching experiments, ${ }^{18}$ since the diffusant is present at very low concentrations and the measurements are performed at temperatures much higher than $T_{g}$. Moreover in our PCS experiments, no dye labeling is required which may affect the diffusing motion. Nevertheless, all three experiments yield $\nu \geqslant 0.6$ for unentangled polymer mixtures. The somewhat higher exponent in the present PCS experiments may imply better screening of the hydrodynamic interactions between PS segments in a mixlure of dissimilar polymers. In the latter, PS diffusion falls between unscreened Zimm coil ( $\nu$ $=0.5)$ and hydrodynamically screened Rouse tube $(\nu=1)$.

\section{B. Dilute ternary mixtures}

For PS/T/PPO and PS/DB/PPO systems, Figs. 1 and 2 display single and double relaxational behavior, respectively, in agreement with the RPA predictions, Eqs. (6) and (7). For the former system, the amplitude $a_{2}^{I}$ and the diffusion coefficient $D_{2}^{\mathrm{I}}$ of the single relaxation process, that is associated with the translational diffusion of PS in the $(\mathrm{PPO}+\mathrm{T}) \mathrm{me-}$ dium, are given by Eqs. (6c) and (6d) for low $\phi_{\mathrm{PS}}$ values, respectively. Equations (5) and (6c) derived under the assumption $\chi_{a c}=\chi_{b c}=0$ predict the same concentration dependence for the diffusion coefficient. Moreover, the former can account for the weak composition dependence of $D$ in PS/PPO blend. Therefore, it is anticipated that the $\phi_{\mathrm{PS}}$ dependence of $D_{2}^{\mathrm{I}}$ is similarly very weak. However, although Eq. (6d) for the $\phi_{\mathrm{PS}}$ dependence of the amplitude is experimentally verified (inset in Fig. 1), the concentration dependence of $D_{2}^{I}$ is found to be unexpectedly strong for low $\phi_{\mathrm{PS}}$. 


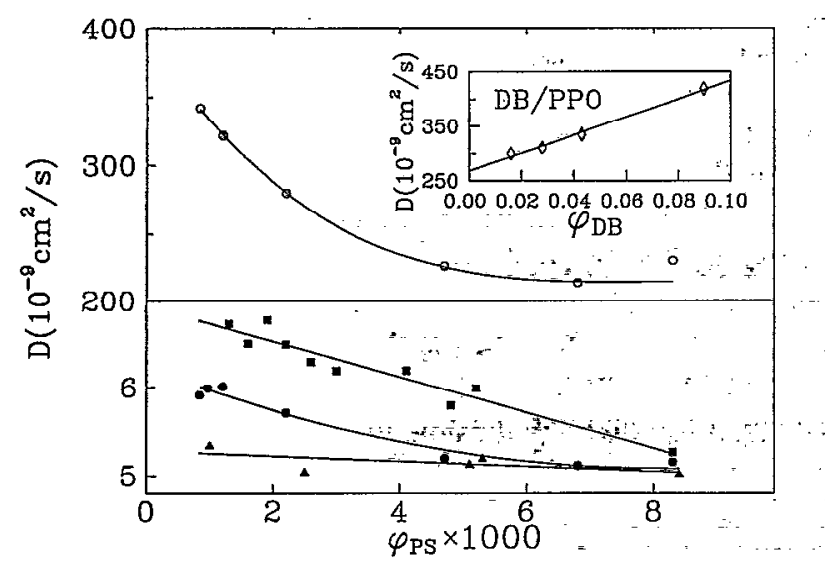

FIG. 5. Polystyrene concentration dependence of the diffusion coefficients in the dilute mixtures PS/PPO $\left(D_{2}, \boldsymbol{\Lambda}\right), \mathrm{PS} / \mathrm{T} / \mathrm{PPO}\left(D_{2}, \boldsymbol{\Pi}\right)$, and the slow $\left(D_{2}, \ominus\right)$, and fast $\left(D_{1}, O\right)$ diffusion coefficient in PS/DB/PPO. The dependence of the diffusion coefficient in the DB/PPO system on DB concentration is shown in the inset $(\diamond)$.

The upturn in the concentration dependence of $D_{2}^{1}$ for $\phi_{\mathrm{PS}}<0.009$ is illustrated in Fig. 5 ; for larger $\phi_{\mathrm{PS}}, D_{2}^{\mathrm{I}}$ coincides within experimental error with $D$ in PS/PPO, also shown in Fig. 5. The presentation of the latter data with Eq. (2) yields $k_{D}=-5.5 \pm 2 \mathrm{~cm}^{3} / \mathrm{g}$. Evidently, the observed variation of $D_{2}^{I}$ with $\phi_{\mathrm{PS}}$ is in disagreement with the predictions of Eq. $(6 \mathrm{c})$.

Strong negative slopes of the concentration dependence of the diffusion coefficients for dilute solutions of high molecular weight homopolymers in theta solvents were usually attributed to unfavorable thermodynamic ințeractions. However, it is very unlikely that the addition of a very small amount of a rather good solvent in the PS/PPO dilute blend with low molecular weight components could affect significantly the thermodynamic forces of the system. Alternatively, the concentration dependence of $D_{2}^{\mathrm{I}}$ may be caused by hydrodynamic forces. The behavior of the second system $\mathrm{PS} / \mathrm{DB} / \mathrm{PPO}$ suggests that the alteration of the PS diffusion at low $\phi_{\mathrm{PS}}$ is not molecular additive specific. For $\phi_{\mathrm{PS}}<0.005$, $D_{2}^{\mathrm{II}}$ exhibits very similar upturn (Fig. 5) in the concentration dependence with that in PS/T/PPO, that is again in disagreement with Eq. (7c). $D_{2}$ in the two ternary systems appears to merge with the diffusion coefficient in the binary mixture at a solvent to polymer segment molar ratio of about 2 for both systems.

The tracer diffusion $D_{\mathrm{PS}}$, obtained from the extrapolated $D_{2}$ 's as $\phi_{\mathrm{PS}} \rightarrow 0$, assumes different values for the three systems, as displayed in Fig. 5. Part of this difference may be explained by the different viscosities, $\eta$, of the three different matrices. The experimental zero-shear bulk viscosities for the three matrices investigated are $439 \pm 3 \mathrm{cP}$ for pure $\mathrm{PPO}$, $399 \pm 3 \mathrm{cP}$ for $\mathrm{PPO} / \mathrm{T}$, and $380 \pm 2 \mathrm{cP}$ for $\mathrm{PPO} / \mathrm{DB}$ with the additive concentrations being the same as in the diffusion measurements. However, the quantity $\left(D_{\mathrm{PS}} \eta\right)$ is not constant as would be expected by the well-known Stokes-Einstein relaxation; in contrast, it varies from $(2.76 \pm 0.15) \times 10^{-8} \mathrm{dyn}$ to $(2.3 \pm 0.2) \times 10^{-8}$ dyn for PS/T/PPO and PS/DB/PPO, respectively, whereas it attains the value of $2.3 \times 10^{-8}$ dyn for

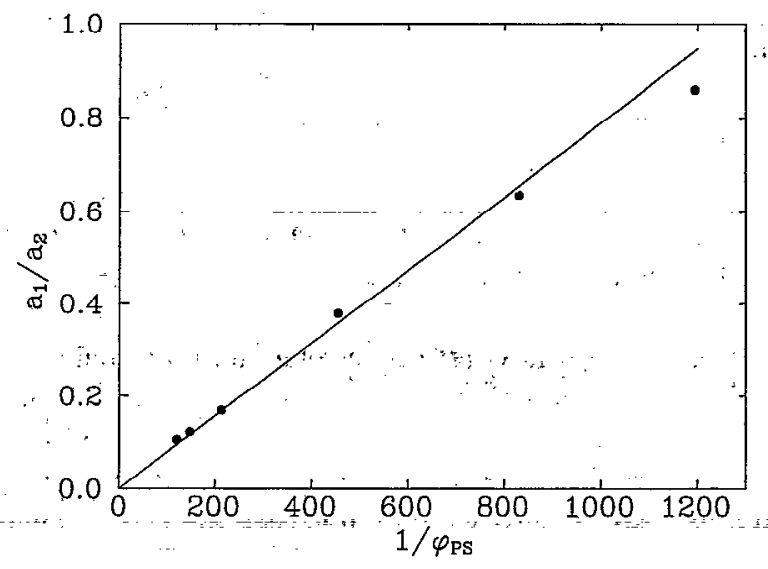

FIG. 6. Polystyrene concentration dependence of the ratio of the experimental amplitudes of the fast to the slow relaxation in PS/DB/PPO dilute ternary blends, plotted according to Eq. (16e).

the binary mixture at $25^{\circ} \mathrm{C}$. Nevertheless, the deviation is significant only for the $\mathrm{T}$ additive (20\% larger), whereas viscosity modification alone may account for the slow mode of the PS/DB/PPO system.

The diffusion coefficient of the fast diffusive relaxation process in PS/DB/PPO is also shown in Fig. 5 as a function of PS concentration. $D_{1}^{\mathrm{m}}$ at low $\phi_{\mathrm{PS}}$ and for low $\phi_{c}$ is related with the translational diffusion $D_{c}$ of DB in the medium of system II, Eq. (7a). The experimental limiting value of $\left(D_{c}^{\mathrm{II}} \eta\right)$ for $\phi_{\mathrm{PS}} \rightarrow 0$ is $135 \times 10^{-8}$ dyn, which is too high for the size of the DB molecule. For comparison, self-diffusion data on comparable size molecules, e.g., benzene, cyclohexane, in liquids yield ${ }^{20}$ significantly lower values $D \eta\left(=15 \times 10^{-8}\right.$ dyn $)$ in closer agreement with the StokesEinstein equation. The variation of $D_{1}^{\mathrm{II}}$ with $\phi_{\mathrm{PS}}$ in Fig. 5 is also much stronger than expected from Eq. (7a), and, moreover, is highly nonlinear; its representation with Eq. (2) is, therefore, inappropriate. Alternatively, the translational diffusion in DB/PPO mixtures increases with DB concentration, as shown in the inset of Fig. 5, and its dependence is described by Eq. (2) with $k_{D}=3.2 \mathrm{~cm}^{3} / \mathrm{g}$.

Figure 6 shows the dependence of the ratio of the amplitudes of the fast to the slow process, $a_{1} / a_{2}$, with the volume fraction of PS. Theory predicts that $a_{1} / a_{2}$ should exhibit a linear dependence on $\phi_{\mathrm{PS}}^{-1}$ with slope almost equal to $\phi_{c} / N_{\mathrm{PS}}$, Eq. (7e); this behavior is experimentally verified in Fig. 6 both with respect to the linear dependence on $1 / \phi_{\mathrm{PS}}$ and for the value of the slope (experimentally $7 \times 10^{-4}$, theory $5 \times 10^{-4}$ ). The verification of this dependence gives more credit to the employed data analysis procedure.

In summary, the discrepancies with the predictions of the RPA theory presented in Sec. II are in the concentration dependence of $D_{1}$ and $D_{2}$ in the presence of a small amount of two different molecular additives. In addition, the observed tracer diffusivities do not absolutely conform to the StokesEinstein relation using the macroscopic shear viscosity.

The concentration dependence of probe diffusion in a wide range of binary and ternary polymer solutions both in the dilute and in the semidilute regimes was recently 
shown ${ }^{21}$ to conform to the hydrodynamic scaling model prediction $^{22}$

$$
D=D^{0} \exp \left(-\alpha c^{\gamma}\right) \text {. }
$$

The parameters $\gamma(0.5 \leqslant \gamma \leqslant 1)$ and $\alpha \sim N^{\delta}$, with $\delta=\mathrm{O}(1)$, are determined by the extent of chain contraction and the strength of hydrodynamic interactions, respectively. At very low concentrations, $\gamma \rightarrow 1$ and Eq. (11) takes the form of Eq. (2) where $k_{D}=-\alpha$ now reflects a retardation of chain diffusion of hydrodynamic nature. The addition of a small amount of a molecular additive decreases the macroscopic viscosity and, hence, can increase $D_{\mathrm{PS}}$ in the limit $\phi_{\mathrm{PS}} \rightarrow 0$. At constant $\phi_{c}$, the increase of $\phi_{\mathrm{PS}}$ would emphasize the role of chainchain interactions that tend to retard the probe chain diffusion. Since interchain penetration in the presence of the additive $\mathrm{C}$ would be different than for the case of a binary mixture $\left(\phi_{c}=0\right)$, the two systems could have different $\alpha$ 's. The diffusion data for the ternary systems in Fig. 5 can alternatively be. described by Eq. (11) with $\gamma=1$ over the whole $\phi_{\mathrm{PS}}$ range; in this case, separation in two concentration regimes is not necessary; $\alpha_{2}=-30 \pm 2$ and $-25 \pm 2$ $\mathrm{cm}^{3} / \mathrm{g}$ for PS/T/PPO and PS/DB/PPO, respectively. The insensitivity of $\alpha$ to the nature of the additive favors hydrodynamic scaling arguments.

Albeit the diffusion of the monomeric solute was not addressed by this scaling model, a fit of the experimental concentration dependence of $D_{1}^{\mathrm{II}}$ yields $\alpha_{1}=55 \pm 5 \mathrm{~cm}^{3} / \mathrm{g}$. The concurrent retardation of PS chain and molecular additive $\mathrm{C}$ diffusion provides support to the role of the hydrodynamic forces. Modification of both rotational and translational diffusion coefficients of molecular solvents by addition of polymeric solute has been experimentally observed ${ }^{23}$ and discussed theoretically. ${ }^{24}$ Taking into account the gradient in the solvent velocity in the vicinity of a polymer bead, the rate of change of the solvent mobility with concentration corresponds roughly to the intrinsic viscosity. The concentration dependence of $D_{1}$ and $D_{2}$ in Fig. 5, expressed in the parameters $\alpha_{1}$ and $\alpha_{2}$, exceed, however, the rate of change in the solution viscosity.

The RPA calculation of the intermediate structure factor, Eq. (3), in ternary polymer systems was based on the Rouse dynamics neglecting hydrodynamic interactions between moving species. However, this assumption may affect the two decay rates $\lambda_{1}$ and $\lambda_{2}$, since, in principle, fluctuating hydrodynamic forces become important in dilute and semidilute solutions. Hydrodynamic interactions were very recently and in the final stages of this work incorporated ${ }^{25}$ in the RPA description of ternary polymer solutions via a concentration blob concept. At intermediate concentrations and in addition to the Rouse contribution, the mobility matrix contains a convolution of the longitudinal component of the Oseen tensor along the $\mathbf{q}$ direction and the static structure factor matrix (Kubo formula). Analytical expressions for the relaxation rates $\lambda_{1}$ and $\lambda_{2}$ are, thcrcfore, precluded; instead, a number of graphical presentations regarding the concentration dependence of $\lambda_{1}$ and $\lambda_{2}$ were generated. ${ }^{25}$ For semidilute solutions, the fast decay rate, $\lambda_{1}$, is related with the cooperative diffusion, whereas $\lambda_{2}$ is assigned to the interdiffusion, as in the present case. The initial variation of $\lambda_{2} / q^{2}$ with polymer volume fraction $\phi$ deviates from the weak linear dependence of Eqs. (6c) and (7c), see, e.g., Fig. 5 of Ref. 25. For low $\phi, \lambda_{2} / q^{2}$ is a decreasing function of $\phi$ and asymptotically approaches the curves without hydrodynamic interactions. This upturn at low $\phi$ depends on the interactions between the two different polymers and the solvent as well as the solvent and solution viscosities. In this framework, quantitative differences in the behavior of $\lambda_{2} / q^{2}$ are conceivable when different additives are used (Fig. 5).

Finally, a pertinent feature is the conformity to the Stokes-Einstein relation using the macroscopic shear viscosity. In PS/T/PPO, $D_{2}^{\mathrm{I}} \eta$ appears to be about $20 \%$ higher than for the other two systems. In the former, a somewhat faster diffusion is conceivable since an optical mismatching between $\mathrm{PPO}$ and $\mathrm{T}$ is possible. In the case of the PS/DB/PPO systems, however, validity of Stokes-Einstein relation for $D_{1}^{\mathrm{II}}$ for the molecular additive would lead to physically unreasonably small size of DB. This may be explained as being due to the fact that in these systems the PPO matrix imposes a smaller viscous drag on the diffusion of the $\mathrm{DB}$ molecules due to the slower polymer relaxation.

\section{CONCLUDING REMARKS}

Photon correlation spectroscopy has been utilized in order to investigate the diffusional dynamics in very dilute PS/ PPO mixtures. Two important advantages of the system are the significant refractive index difference and the fact that the PPO dynamics does not interfere with the measurements. The PS tracer diffusion coefficient is found to scale as $N_{\mathrm{PS}}^{-0.8 \pm 0.04}$, a behavior between the nondraining unscreened Zimm and the free-draining hydrodynamically screened Rouse behavior. The sensitivity of the PS translational diffusion to the presence of a third component even at low concentrations and the possible cffect of hydrodynamic interactions were investigated by extending the measurements to two ternary systems with a low molecular weight component isorefractive either with the matrix PPO or with the minority solute PS chains. The same systems were also treated by an extension of the random phase approximation for the intermediate structure factor in the absence of hydrodynamic interactions, and the new results were discussed in relation to the theoretical predictions. Theory qualitatively captures the pertinent features of the experimental observations, whereas deviations from the theoretical predictions are attributed to increasing effects of hydrodynamic interactions in the ternary systems.

\section{ACKNOWLEDGMENTS}

G.F. acknowledges partial support of the Greek General Secretariat of Research and Technology (Grant under the Operational Programme on Science and Technology). S.H.A. would like to acknowledge that part of this research was sponsored by NATO's Scientific Affairs Division in the framework of the Science for Stability Program.

${ }^{1}$ For a general review see J.-F. Joanny and S. J. Candau, in Comprehensive Polymer Science (Pergamon, Oxford, 1989), Vol. 2, Chap. 7, p. 199.

${ }^{2}$ C. J. C. Edwards, A. Kaye, and R. F. T. Stepto, Macromolecules 17, 773 (1984). 
${ }^{3}$ K. Huber, S. Bantle, P. Lutz, and W. Burchard, Macromolecules 18, 1461 (1985).

${ }^{4}$ A. Sariban and K. Binder, Macromol. Chem. 189, 2357 (1988).

${ }^{5} \mathrm{~K}$. Binder and H. Sillescu, in Encyclopedia of Polymer Science and Engineering, 2nd ed., edited by J. I. Kroschwitz (Wiley, London, 1988), Vol, 2 , p. 1.

${ }^{6} \mathrm{G}$. Meier, G. Fytas, G. Fleischer, and B. Momper, Macromolecules 26, 5310 (1993), and references cited therein.

${ }^{7}$ J. Kanetakis and G. Fytas, J. Chem. Phys. 87, 5048 (1987). G. Meier, B. Momper, and E. W. Fischer, ibid. 97, 5884 (1992).

${ }^{8}$ A. K. Rizos and G. Fytas, Macromolecules 22, 4136 (1989).

${ }^{9}$ M. M. Rodrigez and C. Cohen, Macromolecules 21, 2091 (1988).

${ }^{10} \mathrm{CRC}$ Handbook of Chemistry and Physics, edited by R. C. Weast, 64th ed. (Chemical Rubber, Boca Raton, FL, 1983).

${ }^{1}$ W. J. Huang, T. S. Frick, M. R. Landry, J. A. Lee, T. P. Lodge, and M. Tirrell, AIChE J. 33, 573 (1987).

${ }^{12}$ R. Borsali, M. Duval, H. Benoit, and M. Benmouna, Macromolecules 20, 1112 (1987); L. Giebel, M. Benmouna, R. Borsali, and E. W. Fischer, ibid. 26, 2433 (1993).

${ }^{13}$ P. J. Daivis, D. N. Pinder, and P. T. Callaghan, Macromolecules 25, 170 (1992).
${ }^{14}$ A. Z. Akcasu, B. Hammouda, T. P. Lodge, and C. C. Han, Macromolecules 17, 759 (1984); A. Z. Akcasu, in Dynamic Light Scattering, edited by W. Brown (Oxford University Press, Oxford, 1993), p. 1.

${ }^{15}$ A. Z. Akcasu, M. Benmouna, and H. Benoit, Polymer 27, 1935 (1986).

${ }^{16}$ M. Benmouna, H. Benoit, M. Duval, and A. Z. Akcasu, Macromolecules 20, 1107 (1987); M. Benmouna, M. Duval, and R. Borsali, ibid. 21, 520 (1988).

${ }^{17}$ A. Z. Akcasu and M. Tombakoglu, Macromolecules 23, 607 (1990); A. Z. Akcasu, ibid. 24, 2109 (1991).

${ }^{18}$ B. A. Smith, E. T. Samulski, L. P. Yu, and M. A. Winnik, Phys. Rev. Lett. 52, 45 (1984); Macromolecules 18, 1901 (1985).

${ }^{19}$ P. F. Green and E. J. Kramer, Macromolecules 19, 1108 (1986).

${ }^{20}$ K. J. Czworniqk, H. C. Anderson, and R. Pecora, J. Chem. Phys. 11, 451 (1975).

${ }^{21}$ G. D. J. Phillies, J. Phys. Chem. 93, 5029 (1989).

${ }^{22}$ G. D. J. Phillies, Macromolecules 20, 558 (1987); 21, 3101 (1988).

${ }^{23}$ G. Fytas, A. K. Rizos; and T. P. Lodge, J. Chem. Phys. 93, 5096 (1990); T. P. Lodge, J. Phys. Chem. 97, 1480 (1993).

${ }^{24}$ M. Fixman, J. Chem. Phys. 92, 6858 (1990).

${ }^{25}$ B. Hammouda, Macromolecules 26, 4800 (1993). 